NOTA CIENTÍFICA

\title{
Primer registro de Crepidobothrium gerrardii (Cestoda: Proteocephalidae) en el Perú
}

\author{
First record of Crepidobothrium gerrardii (Cestoda: Proteocephalidae) in \\ Peru
}

\section{Luis A. Gomez-Puerta}

Laboratorio de Medicina Veterinaria Preventiva. Facultad de Medicina Veterinaria. Universidad Nacional Mayor de San Marcos. Av. Circunvalación 2800, San Borja. Lima, Perú.

E-mail: lucho92@yahoo.com

Presentado: $\quad$ 04/05/2011

Aceptado: $\quad 17 / 10 / 2011$

Publicado online: $08 / 02 / 2012$

\section{Resumen}

Se registra por primera vez la presencia en el Perú del cestodo Crepidobothrium gerrardii parasitando el intestino de una boa constrictora (Boa constrictor) procedente del departamento de Loreto. Cuatro especímenes fueron estudiados e identificados como C. gerrardii.

Palabras clave: Crepidobothrium gerrardii, cestodo, Boa constrictor, Perú.

\section{Abstract}

It is the first record of cestode Crepidobothrium gerrardii in Peru, parasitizing the intestine of a boa constrictor (Boa constrictor) from Loreto. Four tapeworms were studied and identified as C. gerrardii.

Keywords: Crepidobothrium gerrardii, cestode, Boa constrictor, Peru.

\section{Introducción}

El grupo de cestodos más destacable en serpientes es la familia Proteocephalidae La Rue, 1911, encontrada en una lista amplia de hospederos del viejo y nuevo mundo (Freze 1965, Rego 1994, Ernst y Ernst 2006). Dentro de esta familia, el género Crepidobothrium Monticelli, 1900; parasita únicamente a serpientes sudamericanas (Rego 1994).

Actualmente, los registros sobre cestodos de serpientes peruanas son escasos, únicamente es conocida Ophiotaenia sp. parasitando a las serpientes Bothrops atrox Linnaeus, 1758, Boa constrictor Linnaeus, 1758, Epicrates cenchria Linnaeus, 1758 y Corallus caninus Linnaeus, 1758(Tantaléan y Gozalo 1985, Sánchez et al. 2004). En el presente trabajo se reporta a Crepidobothrium gerrardii Baird, 1860 como nuevo cestodo para la fauna parasitaria de Perú.

\section{Material y métodos}

En Mayo del 2004, se examinó el sistema digestivo de una boa constrictora (B. constrictor) procedente de la localidad de Nauta (4²9'09.00"S - 733 3'40.96”W), provincia de Iquitos, Loreto, en la amazonía del Perú. Se colectaron 4 cestodos, los cuales fueron fijados y preservados en etanol al 70\%. Para el estudio taxonómico, los cestodos fueron teñidos con acetocarmin ferrico, clarificados en eugenol y montados en Bálsamo de Canadá (Georgiev et al. 1986). Las medidas y fotografías se realizaron en un microscopio Carl Zeiss Axioskop 40, equipado con un ocular micrométrico. Las medidas se expresan como rango en milímetros.

Para la identificación se utilizaron las claves morfológicas propuestas por Rego (1994) y de Chambrier (1989a). La nomenclatura taxonómica se realizo de acuerdo a Rego (2003). Parte de las muestras examinadas se encuentran depositadas en la Colección Helmintológica y de Invertebrados Relacionados del Museo de Historia Natural de la UNMSM (MUSM 2978) Lima, Perú.

\section{Resultados \\ Clase: Cestoda \\ Orden: Proteocephalidea Mola, 1928 \\ FAmilia: Proteocephalidae La Rue, 1911 \\ Género: Crepidobothrium Monticelli, 1900 \\ Crepidobothrium gerrardii Baird, 1860}

Cestode de 320 - $540 \mathrm{~mm}$ de largo con un ancho máximo de $4 \mathrm{~mm}$. El escolex presenta cuatro ventosas prominentes que miden de 0,60-0,72 mm de diámetro. Los proglotidos maduros (Figura 1) miden de 2500 - $4800 \mathrm{~mm}$ de largo por 2450 - 3000 $\mathrm{mm}$ de ancho. Los proglotidos grávidos miden de 4020 - 4750 $\mathrm{mm}$ de largo por $1420-1680 \mathrm{~mm}$ de ancho. El poro genital es irregularmente alternado. Los testículos son numerosos (312 360 ), se encuentran agrupados en dos filas laterales. La vagina se encuentra posterior al saco del cirro. El ovario es bilobulado con un ancho que oscila de 0,99-1,30 mm de largo y se encuentra

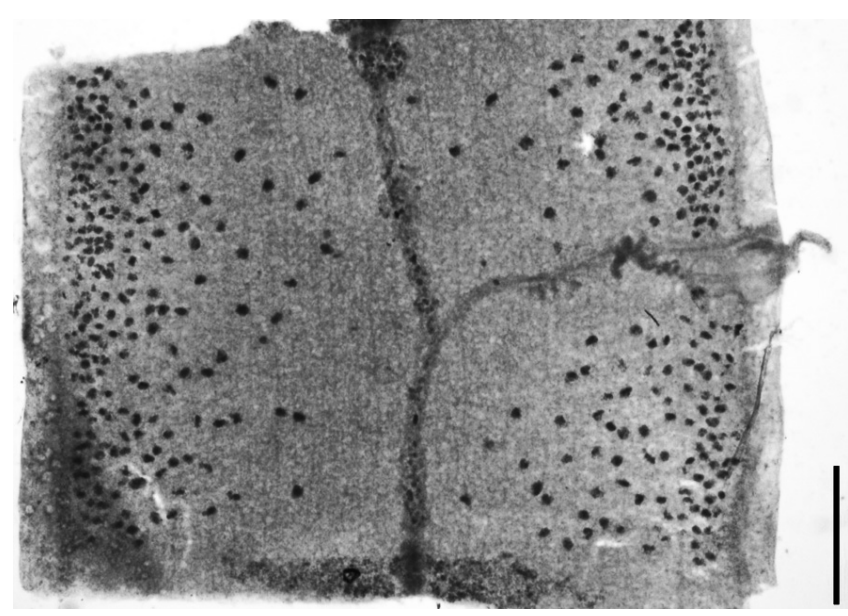

Figura 1. Proglotis maduro de Crepidobothrium gerrardii. Escala $0,5 \mathrm{~mm}$. 
localizado en el margen posterior de los proglotis. Los proglotis grávidos presentan el útero medial con ramas laterales.

Coincidiendo con las descripciones de Chambrier (1989a) concluimos que la especie corresponde a C. gerrardii.

\section{Discusión}

Durante muchos ańos las especies correspondientes al género Crepidobothrium han tenido controversias en lo que respecta a su nomenclatura. Meggitt (1927) considera que el género Crepidobothrium se encuentra conformado por más de 7 especies. Freze (1965) considera cuatro especies para este género. Schmidt (1986) menciona que el género está conformado por solo 5 especies. Finalmente, de Chambrier en 1989 (a y b), realizó una revisión del género Crepidobothrium concluyendo que está representado por 5 especies validas: $C$. gerrardii para la boa constrictora; $C$. dollusi y $C$. lachesidis parásitos de la anaconda (Eunectes murinus Linnaeus, 1758); C. viperis y C. garzonii para Bothrops alternatus Duméril, Bibron \& Duméril, 1854 (de Chambrier 1988, de Chambrier 1989a, de Chambrier 1989b).

Crepidobothrium gerrardii fue descrita por vez primera por Baird (1860) parasitando una boa constrictora procedente de Sudamérica. Posteriormente fueron descritas otras especies de cestodos Proteocephalideos para la boa constrictora. MacCallum (1921) describe a Tetrabothrius boiae parasitando a una $B$. constrictor proveniente de Brasil y a Tetrabothrius brevis para una B. constrictor de México. De Chambrier (1988 a y b) realizó una revisión del material tipo de estos especímenes y concluye que T. boiae y $T$. brevis son sinónimos de $C$. gerrardii.

La distribución de $C$. gerrardii es únicamente para países tropicales (Rego 1967). Los registros en otros países se deben exclusivamente a hallazgos en zoológicos (de Chambrier 1989a). El hallazgo de $C$. gerrardii en Perú permite ampliar el área de distribución del parásito.

\section{Literatura citada}

Baird W. 1860. Description of some new species of intestinal worms (entozoa) in the collection of the British Museum. Proc. Zool. Soc. Lond. 28: 446-448. de Chambrier A. 1988. Crepidobothrium garzonii n. sp. (Cestoda: Proteocephalidae) parasite de Bothrops alternatus Dum. Bibr. \& Dum., 1854 (Serpentes: Viperidae) au Paraguay. Rev. Suisse Zool. 95: 1163-1170.

de Chambrier A. 1989a. Revision du genre Crepidobothrium Monticelli, 1900 (Cestoda: Proteocephalidae) parasite d'Ophidiens néotropicaux. I. C. gerrardii (Baird, 1860) et C. viperis (Beddard, 1913). Rev. Suisse Zool. 96: 191-217.

de Chambrier A. 1989b. Revision du genre Crepidobothrium Monticelli, 1900 (Cestoda; Proteocephalidae) parasite d'Ophidiens néotropicaux. II. C. dollfusi Freze, 1965, C. lachesidis (MacCallum, 1921) et conclusions. Rev. Suisse Zool. 96: 345-380.

Ernst C.H. \& E.M. Ernst. 2006. Synopsis of helminthes endoparasitic in snakes of the United States and Canada. Society for the study of amphibians and reptiles. Herpetological Survival No $34.86 \mathrm{pp}$

Freze V.I. 1965. Essentials of cestodology, vol. V. Proteocephalata in fish, amphibians and reptiles. Nauka, Moscow, 538 p. (In Russian: English translation, Israel Program of Scientific Translation.

Georgiev B., V. Biserkov \& T. Genov. 1986. In toto staining method for cestodes with iron acetocarmine. Helminthologia. 23: 279-281.

MacCallum G.A. 1921. Studies in helminthology. Zoopathologica. 1: $137-284$

Meggitt F.J. 1927. Remarks on the Cestode families Monticellidae and Ichthyotaeniidae. Ann. Trop. Med. Parasitol. 21: 69-87

Rego A.A. 1967. Sobre alguns cestódeos parasitos de répteis. Rev. Brasil. Biol. 27: 181-187.

Rego A.A. 1994. The order Proteocephalidea. In Keys to the cestode parasites of vertebrates, L. Khalil, A. Jones, and R.A. Bray (eds.). Commonwealth Agricultural Bureaux, Wallingford, U.K., p. 257-293.

Rego A.A. 2003. Problems of classification of South American Proteocephalids (Cestoda). On a new classification for the group. Acta Scientiarum, 25 (1) : 15-22.

Sánchez N., M. Tantaleán, R. Richards \& H. Gálvez. 2004. Parásitos helmintos en Boa constrictor, Epicrates cenchria y Corallus caninus (Ophidia: Boidae) criadas en cautiverio. Rev. Inv. Vet. Perú. 15: 166-169.

Schmidt G.D. 1986. Handbook of tapeworm identification. CRC Press, Boca Raton, Florida.

Tantaléan, M. \& A. Gozalo. 1985. Parásitos de Bothrops atrox (Viperidae) de la Amazonía peruana. AMVEAP. 20: 11-12. 